\title{
The Nature of Shadows, from Yale to Bilkent
}

\author{
ISTVÁN ARANYOSI
}

\begin{abstract}
I discuss a solution to the Yale shadow puzzle, due to Roy Sorensen, based on the actual process theory of causation, and argue that it does not work in the case of a new version of the puzzle, which I call 'the Bilkent shadow puzzle'. I offer a picture of the ontology of shadows which, together with an alternative view of causation, constitutes the basis for a new solution that uniformly solves both puzzles.
\end{abstract}

The Yale shadow puzzle ${ }^{1}$ purports to involve the ultimate incompatibility of the following plausible looking principles:

(2) Shadows cannot be cast through an opaque object.

(3) Every shadow is the shadow of something.

Imagine a high wall casting a shadow on the ground on a sunny day, and a little bird standing on the ground within the shadow cast by the wall. Is the shadow that is present on the ground under the bird-let's mark it with $S$ - the shadow of the bird, or (part of) the shadow of the wall? It cannot be the bird's, by proposition (1), as the bird does not block any light. Neither can it be the wall's, by proposition (2), as the wall cannot cast its shadow through the opaque bird. Yet, by proposition (3), it has to be the shadow of something.

Roy Sorensen has offered neat looking solution to the puzzle; however, part of my goal in this paper is to show that this solution doesn't work for a new version of the Yale shadow puzzle, which I call 'The Bilkent shadow puzzle', in acknowledgment of my own

1 Named so because it used to be a common lunchtime discussion topic among Yale University philosophers in the 1970s. Its first mention and discussion in print, to my knowledge, is in S.Todes and C. Daniels, 'Beyond the doubt of a shadow: a phenomenological and linguistic analysis of shadows', Selected Studies in Phenomenology and Existential Philosophy $\mathbf{5}$ (1975), 203-216. It has subsequently been discussed by Bas Van Fraassen in Laws and Symmetry (New York and Oxford: Oxford University Press, 1989, 217-9) and by Roy Sorensen in Seeing Dark Things. The Philosophy of Shadows (New York and Oxford: Oxford University Press, 2008, 52-54 and 98-99). 


\section{István Aranyosi}

academic affiliation. The remaining issue will be to offer a new solution that would cover both puzzles. As it will turn out, the solution is based on a peculiar understanding of the ontology of shadows.

\section{A solution to the Yale puzzle}

Sorensen's solution to the Yale shadow puzzle is very simple and elegant; let us quote him²:

Principle (2) [i.e. our proposition (2), n.a.] is true but insinuates that the only way that a shadow can appear on the far side of an intervening object is by penetration. It discourages us from considering the alternative that the shadow appears on the far side by default. Nothing aside from the original blockage of the light is needed to place shadow there.

According to Sorensen's proposal, therefore, the shadow that falls under the bird is the shadow of the wall, as it is the wall that blocks light, and its casting a shadow does not count as 'casting it through the bird'. Blocking the light is, by default, sufficient for casting a shadow. Hence, Sorensen calls his solution 'the blockage theory'.

Further, this solution is in line with Sorensen's general commitment throughout his book to rejecting the counterfactual theory of causation due to David Lewis ${ }^{3}$, and subscribing to the 'actual physical process' view of it, according to which what counts in causation is not what would be the case but the actual physical process that does it. In the Yale puzzle, then, the actual physical process that is responsible for darkness to be present on the far side of the wall is the light-blocking process due to the interaction between light and the wall.

\section{The Bilkent shadow puzzle}

With a very small change effected on the original Yale puzzle we get the Bilkent puzzle, which, given that it is structurally the same as the original, is damaging to Sorensen's solution explained in the previous section. All we need to do is to swap the positions of the bird and the wall. Instead of the bird being located within the shadow of the wall, it will now fly above the wall, closer to the sun, thus casting a shadow
Op. cit. note 1, 53.
D. Lewis, 'Causation', Fournal of Philosophy 70 (1973), 556-67. 
on the wall. The question is the same: is the dark patch on the ground, call it $S^{*}$, on the far and dark side of the wall, that is in line with the bird, (part of) the shadow of the wall, or the shadow of the bird?

By (2) it can't be the bird's shadow, as it would thus be cast through the opaque wall; by (1) it can't be the shadow of the wall either, as the relevant portion of the wall is already shaded by the bird, so it doesn't block any light; yet, it has to be the shadow of something, by (3).

The new puzzle is damaging to Sorensen's solution to the Yale puzzle. If Sorensen's blockage theory were right, we would have to say that since it is the bird that blocks the relevant quantity of light, $S^{*}$ is the shadow of the bird. But this can't be the case because in the new puzzle $S^{*}$ would really count as being cast through the wall - the bird's shadow is actually cast on the wall; it 'stops there' as it were!

Further, given the actual physical process view of causation that Sorensen champions, since the relevant part of the wall is already shaded by the bird, so that it can't be part of the light-blocking physical process, it is again the bird that has to be taken as the shadow caster of $S^{*}$, which seems clearly wrong - by analogy, we should say that the reason it is dark at $1 \mathrm{a} . \mathrm{m}$. in Yale (New Haven, US), when there is a huge cloud over Bilkent (Ankara, Turkey) at 7 a.m., is that Yale is in the shadow of that cloud. ${ }^{4}$

\section{Influence and exstitution}

The solution to both puzzles is based on the recognition that the question about shadows $S$ and $S^{*}$ is ill posed, as they are not actually shadows. Just like subregions corresponding to proper parts of holes are not themselves holes, subregions corresponding to proper parts of shadows are not themselves shadows; and if this is so, principles (1)-(3) are accommodated in virtue of neither S, nor $\mathrm{S}^{*}$ counting as being in the category of shadows. Still, there is a question as about the entities that do fall under the heading of shadows. This brings me to what in earlier work I put forward under the name of 'material exstitution view' (MEV) of shadows, as opposed to the 'immaterial constitution view' $(\mathrm{ICV})^{5}$ :

\footnotetext{
There is a six hour time zone difference between Yale and Bilkent.

I. Aranyosi, 'Shadows of constitution', Monist 90 (2007), 415-431 (quote from 417-8).
} 


\section{István Aranyosi}

According to ICV, shadows are standard spatial entities hosted by material surfaces, just like regions of material surfaces themselves, except that they are not composed of matter, but from missing light. (5) They are immaterially constituted by subregions of the region they cover. The mereology of shadows as so conceived is no different from the standard mereology of concrete material individuals. (...)

(...) Let us turn to MEV. According to this view shadows are spatially determined not by immaterial parts present in the subregions of the region they occupy, but by extraneous light, that is, by the configuration of light that delimits the region occupied by them. In this sense shadows are not constituted, but they are what we may call 'exstituted', determined by a certain collection of materially occupied regions outside them.

If we adopt the material exstitution view, then neither $S$, nor $S^{*}$ count as shadows. The only shadow in the Yale puzzle is the one that is surrounded by the light that has not been blocked by the wall. In the Bilkent puzzle there are two shadows the one that is cast by the bird on the wall and the one that is delimited by the light that has not been blocked by the wall. In both cases, therefore, the dark region on the far side of the wall is the shadow of the wall.

The problem is, still, that the counterfactual theory does not sanction these conclusions: in both puzzles it is false that had the wall been removed, the whole shadow would have disappeared. So it looks as though both the actual process theory and the counterfactual theory are unsatisfactory when it comes to addressing the puzzles. However, in other earlier work on shadows ${ }^{6} \mathrm{I}$ argued for Lewis's latest counterfactual theory of causation - causation as influence as relevant in shadow related puzzles. According to this theory, $C$ causes $E$ iff a series of changes in $E$ counterfactually depend on a corresponding series of small alterations in $C .^{7}$ The notion of influence neatly explains why the shadow of the wall is exclusively to be found in both puzzles on the far side of the wall. It is because the wall alone exerts influence on the shadows in both puzzles. Small changes in the position or size of the wall will generate small changes in the

6 In I. Aranyosi, 'Review of Roy Sorensen, Seeing Dark Things. The Philosophy of Shadows (Oxford University Press, 2008)', Australasian Fournal of Philosophy 86 (2008), 513-515 (on page 514), and in I. Aranyosi, 'The reappearing act', Acta Analytica 24 (2009), 1-10 (on page 7).

David Lewis, 'Causation as influence', Fournal of Philosophy 97 (2000), 182-97 (on 188-190). 
configuration of light surrounding the shadow. Not so for the bird small changes in the position or size of the bird will have no influence whatsoever on the light-darkness configuration on the far side of the wall.

To conclude, in both the Yale and the Bilkent shadow puzzle principles (1)-(3) can be accommodated by adopting (a) the view that shadows are determined by extraneous light, so that proper parts of shadows are not themselves shadows, and (b) the view that shadows are cast by objects that have influence, in Lewis's sense, on them. ${ }^{8}$

Bilkent University, Turkey aranyosi@bilkent.edu.tr

8 For discussion about the puzzles I would like to thank Ezgi Ulusoy Aranyosi. I would also like to dedicate this paper to the memory of Ihsan Doğramaci, founder and chairman of the Board of Trustees of Bilkent University. 